\title{
Combined approach for malignant tumours of the deep lobe of the parotid involving the stylomandibular tunnel and parapharyngeal
} space

\author{
Narayana Subramaniam ${ }^{1}$, Carsten Palme ${ }^{1}$, and Tsu-Hui (Hubert) Low ${ }^{1}$ \\ ${ }^{1}$ Chris O’Brien Lifehouse
}

August 20, 2020

\begin{abstract}
En-bloc clearance of malignant deep lobe parotid tumours involving the stylomandibular tunnel and parapharyngeal space is challenging in view of the limited surgical access and the critical structures that are located in close proximity The technique that we describe is a combined transmandibular-transmastoid approach that allows complete en-bloc resection of the deep parotid lobe and stylomandibular tunnel with a lateral temporal bone resection and a marginal mandibulectomy This approach provides good exposure of the critical structures and allows for a safe en-bloc resection and good oncological margins This clinical scenario is common in Australia, due to the high incidence of cutaneous squamous cell carcinoma involving the parotid gland, either through direct invasion or lymphatic spread In this article we explain step-by-step how to perform these resections to allow potential cure in patients with very locally advanced disease
\end{abstract}

Combined approach for malignant tumours of the deep lobe of the parotid involving the stylomandibular tunnel and parapharyngeal space

\section{Succinct points}

- En-bloc clearance of malignant deep lobe parotid tumours involving the stylomandibular tunnel and parapharyngeal space is challenging in view of the limited surgical access and the critical structures that are located in close proximity

- The technique that we describe is a combined transmandibular-transmastoid approach that allows complete en-bloc resection of the deep parotid lobe and stylomandibular tunnel with a lateral temporal bone resection and a marginal mandibulectomy

- This approach provides good exposure of the critical structures and allows for a safe en-bloc resection and good oncological margins

- This clinical scenario is common in Australia, due to the high incidence of cutaneous squamous cell carcinoma involving the parotid gland, either through direct invasion or lymphatic spread

- In this article we explain step-by-step how to perform these resections to allow potential cure in patients with very locally advanced disease

\section{Introduction and clinical case}

En-bloc clearance of malignant deep lobe parotid tumours involving the stylomandibular tunnel and parapharyngeal space is challenging in view of the limited surgical access and the critical structures that are located in close proximity (1). As most of the tumours that involve the deep lobe of the parotid gland and the post-styloid parapharyngeal space are benign, they can be removed through blunt dissection with subcapsular dissection technique (2-3). However malignant tumours involving this space require clearance 
with adequate oncological margin; therefore a good exposure of the surrounding structures is paramount, to avoid adverse complications during resection.

The technique that we describe is a combined transmandibular-transmastoid approach that allows complete en-bloc resection of the deep parotid lobe and stylomandibular tunnel with a lateral temporal bone resection and a marginal mandibulectomy. This approach provides good exposure of the critical structures and allows for an en-bloc resection and good oncological margins.

A 75-year-old female patient had a history of cutaneous squamous cell carcinoma in the right lower neck with metastases to the right parotid gland and cervical nodes, undergoing wide excision of the skin lesion, parotidectomy, selective neck dissection and an anterolateral thigh free flap reconstruction fourteen months previously. Four months ago, she presented with a recurrent lesion in the right parotid bed. The contrast enhanced CT head and neck of the patient shows a heterogeneously enhancing area involving the right superficial and deep lobes of the parotid gland with extension through the stylomandibular tunnel into the parapharyngeal space (figure 1). A complete resection would involve a total parotidectomy, segmental mandibulectomy, masticator space clearance and a lateral temporal bone resection with sacrifice of the involved pinna and facial nerve. The technique described below is an approach for similar tumours that allows total en-bloc resection with adequate oncologic margin, in order to maximize the tumour control.

The initial part of the surgery involved a radical parotidectomy approach. The area of skin involved is shown with a dashed line, while the final skin margin is shown with a solid line (figure 2). An extension along the neck was made for completion neck dissection and exposing the neck vessels for the free flap reconstruction. A subplatysmal flap/parotid fascial plane was raised radially with exposure of the entire parotid gland with all the distal branches of the facial nerve delineated for subsequent facial nerve reanimation. The junction of the proximal and middle thirds of the body of the mandible was also exposed to allow an osteotomy as shown.

Next, the lateral temporal bone resection was performed; this involved an initial cortical mastoidectomy with identification of the antrum, lateral canal and incus. Using these landmarks, the mastoid portion of the facial nerve trunk was identified and exposed. The descending portion of facial was transacted with a section sent for frozen section. The distal portion of the remainder of the intra-temporal facial nerve was marked and left in continuity with the rest of the tumour specimen. A posterior tympanotomy was performed, the incudostapedial joint was identified and disarticulated. After removal of the incus, malleus head and division of the tensor tympani, a bone cut was made superiorly along the zygomatic arch to the superior aspect of the TMJ capsule. Another bone cut was made inferiorly, anterior to the facial canal, and superior to the jugular bulb to completely free up the external ear canal.

The facial nerve was then lifted out of the temporal facial canal, with removal of the mastoid tip and freeing of the soft tissue just deep to it. The right external auditory canal was then down-fractured, with en-bloc removal along with the posterior segment of the zygomatic arch and condyle of the mandible.

Finally, before removal of the entire specimen, the mandible osteotomy was retracted to expose the medial pterygoid muscle. The muscle was resected, maintaining a good margin of uninvolved tissue. At this point, the tumour was freed circumferentially with good margins. The tumour was then distracted outwards for adequate resection of the deep margin (figure 3). The final defect is seen in figure 4 .

A primary facial nerve reanimation was performed, with a right brow lift, a lateral canthoplasty, nasolabial lift and cable graft from the proximal facial nerve stump to the midface branches. A chimeric anterolateral thigh flap was used, with skin to reconstruct the skin defect and vastus lateralis muscle to obliterate the mastoid cavity, after plugging of the eustachian tube opening.

The final tumour margins were circumferentially clear, with a deep margin clearance of $10 \mathrm{~mm}$. (figure 5). The post-operative period was uneventful except for a small area of superficial skin loss over the previous flap. In view of the large volume recurrent disease she was administered adjuvant radiotherapy, which she completed four months ago. She is currently disease free. In addition to this case, we have successfully 
performed this procedure in other patients $(n=4)$. It can be reliably repeated in these tumours in a safe manner to achieve adequate oncological clearance.

\section{Discussion}

This technique is an effective combined approach to resect large malignant tumours involving the stylomandibular tunnel, parapharyngeal and masticator spaces with adequate circumferential oncological margins in a safe and controlled manner. Although the stylomandibular tunnel is a narrow area with potential spread to the pre-styloid parapharyngeal space and masticator space, an appropriate mandibulotomy permits sufficient anterior exposure with concurrent lateral temporal bone resection and enough posterior exposure to allow malignant tumours in this region to be removed safely.

This clinical scenario is common in Australia, due to the high incidence of cutaneous squamous cell carcinoma involving the parotid gland, either through direct invasion or lymphatic spread (4). Similarly, this technique can be utilized in malignant primary parotid lesions that have extended through the stylomandibular tunnel, into the parapharyngeal and masticator spaces.

Most of these malignant tumours would either present with facial nerve weakness or encasement of some component of the facial nerve, necessitating a concurrent resection of the facial nerve and reconstruction wherever possible.

\section{References}

1. Shahab R, Heliwell T, Jones AS. How we do it: a series of 114 primary pharyngeal space neoplasms. Clin Otolaryngol 2005;30(4):364-7.

2. Cohen SM, Burkey BB, Netterville JL. Surgical management of parapharyngeal space masses. Head Neck 2005;27(8):669-75.

3. Riffat F, Dwivedi RC, Palme C, Fish B, Jani P. A systematic review of 1143 parapharyngeal space tumors reported over 20 years. Oral oncology. 2014 May 1;50(5):421-30.

4. Kelder W, Ebrahimi A, Forest VI, Gao K, Murali R, Clark JR. Cutaneous head and neck squamous cell carcinoma with regional metastases: the prognostic importance of soft tissue metastases and extranodal spread. Annals of surgical oncology. 2012 Jan 1;19(1):274-9.

\section{Figure legend}

Figure 1: (A) Contrast enhanced CT of the head and neck showed a heterogeneously enhancing lesion in the previous surgical bed with a central area of necrosis. The lesion was located deep to the previous free flap reconstruction and involved the right pinna, superficial and deep parotid lobes, the stylomandibular tunnel and masticator space. It abuts the mandible and masseter anteriorly. The dotted-line wedge shows the extent of resection required (B) Another cross sectional image showing the extent of petrous temporal bone that needs to be resected in order to ensure adequate oncological clearance; without a temporal bone resection the posterior margin cannot be adequate in tumours such as these. The dotted-line wedge shows the extent of temporal bone resection required to get margin adequacy

Figure 2: Pre-operative image shows extent of tumour (inner dashed line) and planned extent of resection (outer solid line) with a linear extent to expose the neck vessels

Figure 3: Final en-bloc resection specimen showing (A) superficial view with pinna, skin, external auditory canal, zygoma and mandible (B) deep view showing deep lobe of parotid gland and masticator space and facial nerve stump marked with a prolene stitch. Deep margin of parapharyngeal space fat as seen in gross (C) and microscopy (D)

Figure 4: Final resection bed showing (A) pinna remnant (B) temporal bone remnant (C) zygoma remnant (D) facial nerve distant stumps marked with prolene sutures (E) mandible margin (F) skin flap (G) common 
carotid artery (H) internal jugular vein (I) sternocleidomastoid muscle $(\mathrm{J})$ parapharyngeal fat which serves as deep margin
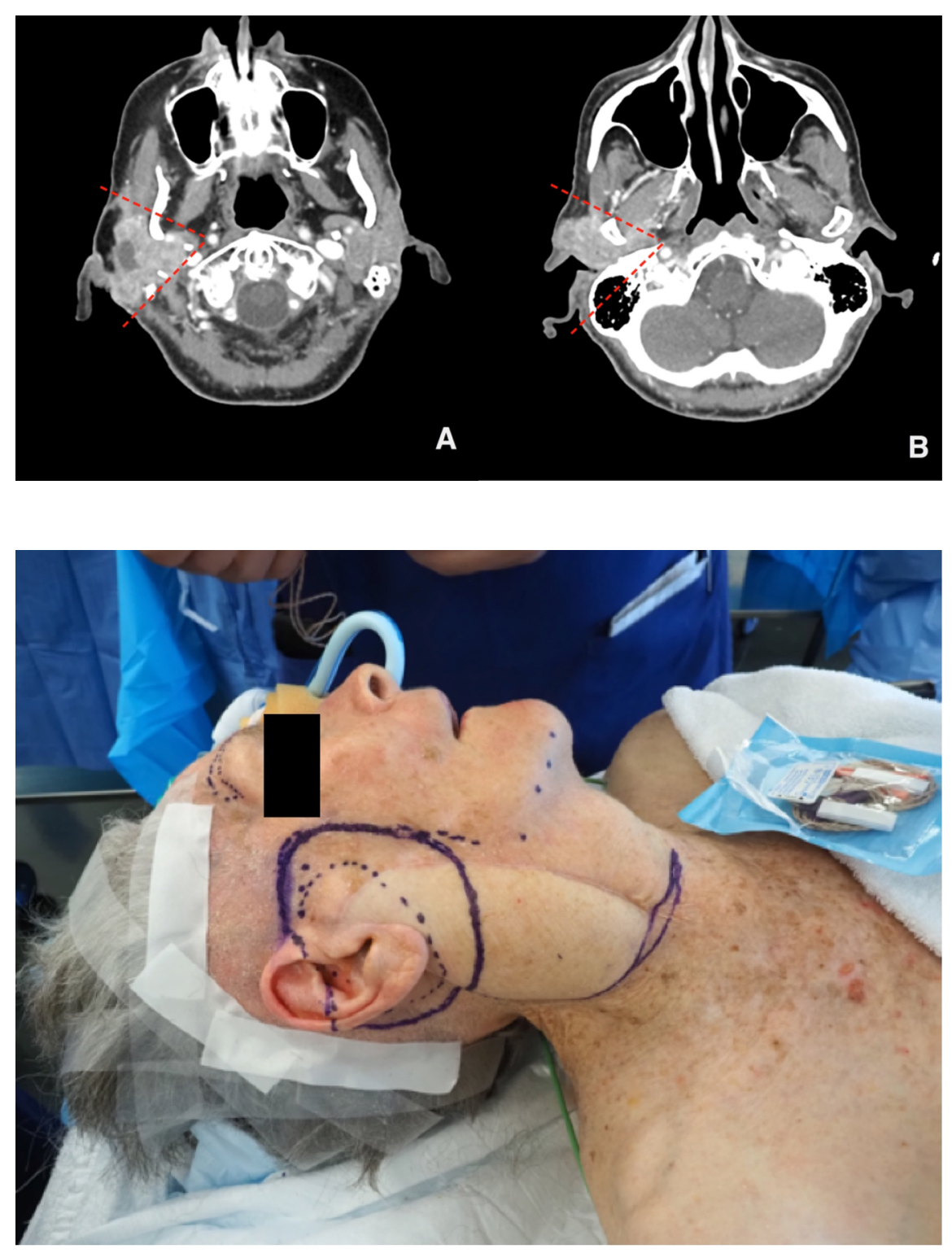

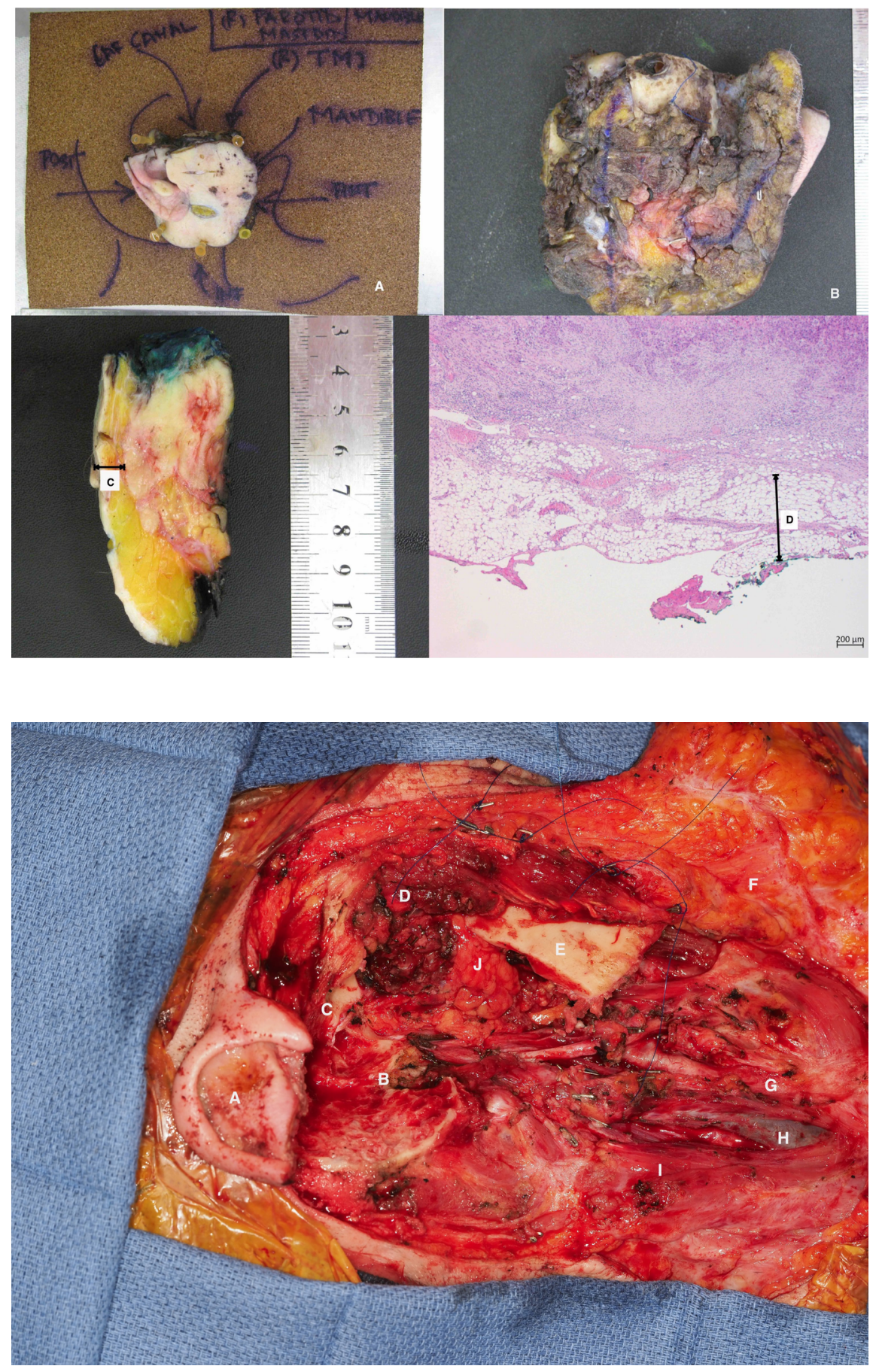